\title{
Quality of Service Enhancement via Compression Technique for Congested Low Bandwidth Network
}

\author{
Ling Sun Tan ${ }^{1}$, Sei Ping Lau ${ }^{2}$, Chong Eng Tan ${ }^{3}$ \\ Faculty of Computer Science \& Information Technology, \\ University Malaysia Sarawak, \\ 94300 Kota Samarahan, Sarawak, Malaysia \\ e-mail: swy41@yahoo.co.uk ${ }^{1}$, splau@fit.unimas.my ${ }^{2}$, cetan@fit.unimas.my ${ }^{3}$
}

\begin{abstract}
Achieving the required Quality of Service (QoS) is an important requirement in a communication network. In recent years, VSAT technology has become one of the popular means to interconnect with people around the world. However, VSAT satellite network has high latency constraint and provides low and limited bandwidth. These limitations caused network congestion when the bandwidth-intensive real-time interactive applications are running over the low bandwidth link. High packet drop incurred by the network congestion leads to degradation of the QoS of the communication link. Therefore, this paper proposes a real-time adaptive packet compression scheme especially for a congested low bandwidth network. This scheme is intended to further enhance the QoS of the low bandwidth network and improve the user experience. Simulation results demonstrate the effectiveness and efficiency of the proposed scheme in enhancing the QoS of the congested low bandwidth network.
\end{abstract}

Keywords-Quality of Service; network congestion; packet compression; low bandwidth

\section{INTRODUCTION}

Quality of Service (QoS) refers to a broad collection of networking technologies and techniques to provide guarantees on the ability of a network to deliver predictable results and enhance user experiences [1]. It is the ability to provide different priority to different applications, users or data flows to guarantee a certain level of network performance. QoS guarantees are crucial especially for those real-time streaming multimedia applications [1]. The characteristics of real-time applications differ significantly from those of non-realtime applications. These real-time applications have higher bandwidth requirements; hence they are referred as bandwidth-extensive applications. Besides, real-time applications are delay sensitive. They require low end-toend delay in order to maintain their interactive and streaming nature. The desired delivery time for each message transmitted across the network is bounded by a specific maximum latency, resulting in a deadline being associated with each message [2]. Examples of these realtime applications include voice over Internet Protocol (VoIP), online interactive games, e-commerce transactions, video conferencing applications, instant messaging and so on.

In recent years, the popularity of real-time interactive applications has been rapidly growing and they have been widely used over cost-effective low bandwidth network such as VSAT (Very Small Aperture Terminal) satellite network. VSATs are a single, flexible communication platform which can be deployed easily and cost efficiently to provide telecommunication solutions for consumers, governments and corporations, even in the remote locations [3]. VSAT satellite network offers value-added satellite-based services capable of supporting the Internet, data, video, LAN, voice and fax communications.

However, most VSAT satellite network provides low and limited network bandwidth and cannot cope with the bandwidth need of real-time applications. Serious network threats like network congestion and packet loss might occur when those real-time interactive applications that consume high bandwidth are running over a low bandwidth satellite link. Under a low bandwidth link, network congestion occurs when the real-time applications are sending more data than the network devices can accommodate. This caused network packets being dropped when the buffers of network devices are filled up and became overflow. Thus, a severely congested link results in an increasing of packet drops, causing the real-time applications to retransmit the data, thereby further increasing the congestion. These result in reduced QoS of the link and also late packet delivery issues, which will create negative impacts on the QoS of the real-time applications as well as user experience. Owing to that, it is necessary to optimize the low bandwidth network to allow better user experience while using the real-time applications.

Therefore, to further enhance the QoS of a congested low bandwidth link, a real-time adaptive packet compression scheme is proposed in prior work [4] to reduce the bandwidth requirement of real-time applications, avoid network congestion and fully optimize the use of the low bandwidth link. In the proposed scheme, compression technique is used to eliminate redundancies in packet in order to overcome the network packet overhead and reduce packet size. Results show that data compression plays a crucial role in improving the QoS of low bandwidth VSAT satellite network. Through data compression, the network load is greatly reduced as more packets can be transmitted over the communication link at one time, hence improving the efficiency of bandwidth utilization and preventing network congestion. In some cases, the efficiency of the bandwidth can be improved up to $259 \%$. By reducing the packet drops caused by network congestion, the QoS of a congested low bandwidth network can be improved significantly. The proposed scheme helps to increase the efficiency and smoothness of real-time traffic over a congested low bandwidth network. 